\title{
On necessary conditions for scale-freedom in complex networks, with applications to computer communication systems
}

\author{
W.J. Xiao ${ }^{\text {a }}$, W.D. Chen ${ }^{\mathrm{a}}$ and B. Parhami ${ }^{\mathrm{b} *}$ \\ ${ }^{a}$ Department of Computer Science, Guangdong Key Laboratory for Computer Networks, South China University of \\ Technology, Guangzhou 510641, P. R. China; ${ }^{b}$ Department of Electrical \& Computer Engineering, University of California, \\ Santa Barbara, CA 93106-9560, USA
}

(Received 6 June 2008; final version received 6 August 2009)

\begin{abstract}
Many complex networks exhibit a scale-free, power-law distribution of vertex degrees. This common feature is a consequence of two generic mechanisms relating to the formation of real networks: (i) networks tend to expand over time through the addition of new vertices and (ii) new vertices attach preferentially to those that are already well connected. We show that for many natural or man-made complex networks possessing a scale-free power-law distribution with the exponent $\gamma \geq 2$, the number of degree-1 vertices, when nonzero, is of the same order as the network size $N$ and that the average degree is of order at most $\log N$. Our results expose another necessary characteristic of such networks. Furthermore, our method has the benefit of relying only on conditions that are static and easily verified for arbitrary networks. We use the preceding results to derive a closed-form formula approximating the distance distribution in scale-free networks. Such distributions are applied extensively in the fields of computer communication and software architecture, among other domains.
\end{abstract}

Keywords: average distance; biological network; complex system; computer network; graph; power-law distribution; scale-free network; small-world network; social network

\section{Introduction}

Complex systems with many components and associated interactions arise in nature, society and many human artefacts. Interactions in such systems can be modelled by networks composed of vertices and links, which are in turn abstracted as undirected or directed graphs. A graph $G$, denoted as $G=(V, E)$, has a set $V$ of vertices or nodes and a set $E$ of edges or links, where each edge is defined by a pair of vertices (ordered pair, for directed graphs). Complex systems in the three categories of natural, societal and synthetic include:

(1) Protein interactions, metabolic systems, contagious diseases

(2) Acquaintances, movie-actor peer group, research collaborators

(3) Power grid, Internet connectivity, Worldwide Web linkages

In citing the above examples, we have limited ourselves to the better known and most widely discussed/studied varieties; many other types of complex networks exist. Such complex networks or graphs are typically neither random (amenable to probabilistic analysis) nor regular (mathematically tractable), rendering the systematic study of their properties a rather challenging undertaking. Many interesting results about such networks have been published over the past decade and many more await discovery. The extreme theoretical and practical interest in such complex networks makes it imperative to develop tools for their recognition and classification.

Two models of complex networks have been studied extensively (Watts and Strogatz 1998; Barabási and Albert 1999; Albert and Barabási 2002; Newman 2003): the small-world model and the scale-free one. The small-world model features localised clusters that are connected by occasional long-range links, leading to an average distance between vertices that grows logarithmically with the network size $N$. Watts and Strogatz (1998) investigated mechanisms via which a regular network can be transformed into a small-world network, without significantly modifying the vertex-degree distribution, and quantified the parameters that characterise the resulting structures.

Scale-free networks, on the other hand, tend to have uneven vertex connectivities, so that a certain fraction of vertices, independent of network size, are highly connected (the hubs). Barabási and Albert (1999) demonstrated that the scale-free power-law distribution of vertex degrees in many large networks is a direct consequence of two generic mechanisms that

\section{*Corresponding author. Email: parhami@ece.ucsb.edu}




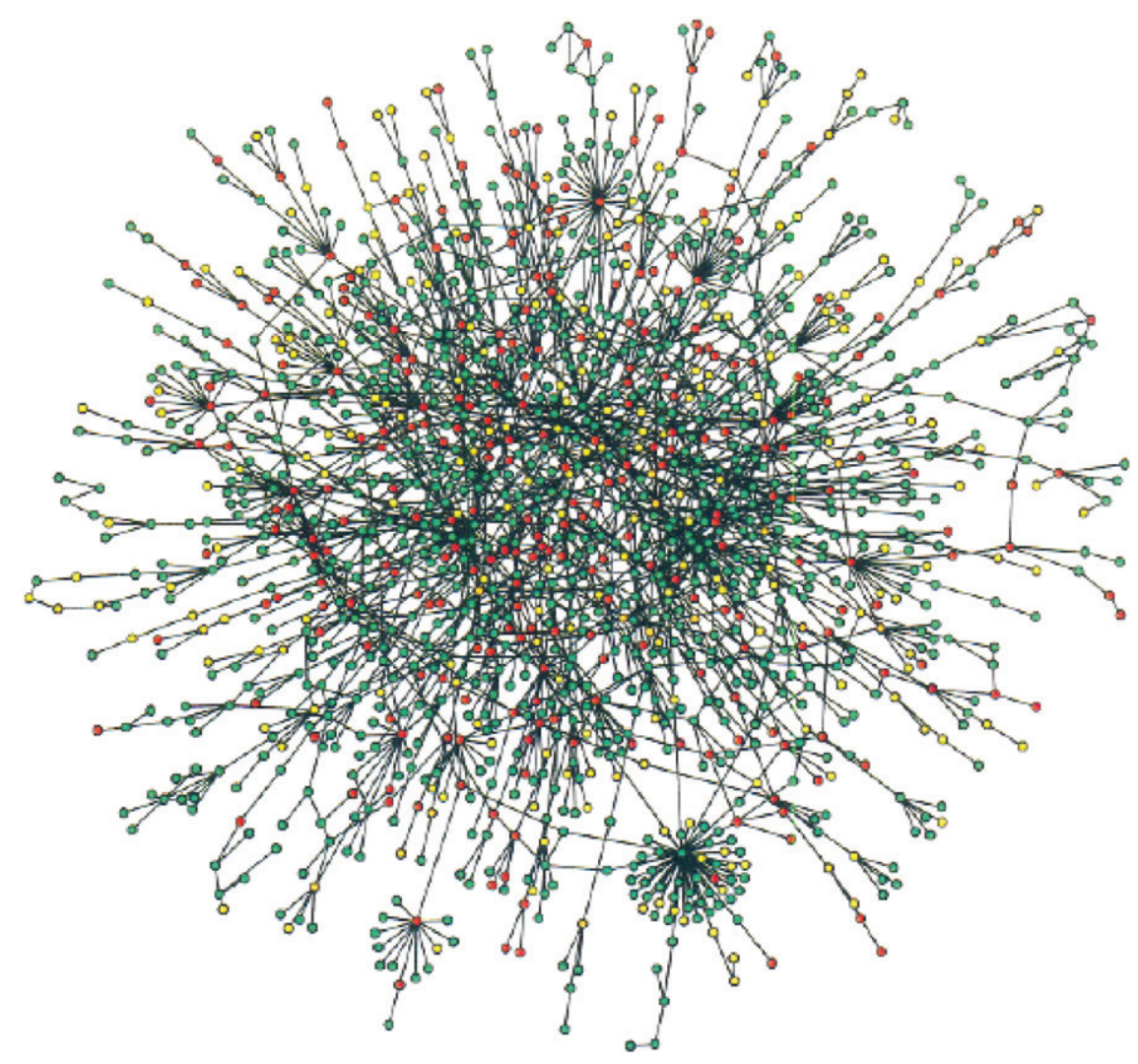

Figure 1. Example scale-free network, representing interacting proteins in yeast (Barabási and Bonabeau 2003).

govern network formation: (i) expansion over time through the addition of new vertices and (ii) preferential attachment of new vertices to those already well connected. It is well known that scale-freedom of a network has significant implications for its diffusion properties and its robustness.

In this article, we focus on scale-free networks, an example of which appears in Figure 1 (Barabási and Bonabeau 2003). We develop analytical tools that help answer the question of whether a network is scale-free. Note that visual representations, of the type shown in Figure 1, reveal much about the structure of a network that may not be readily discerned from a list of nodes and links. We will return to discuss our motivation after we have introduced some needed background in Section 2.

\section{Background and motivation}

Despite some similarities, small-world and scale-free networks have key differences. They both reside at the boundary between regularity and randomness. However, small-world networks tend to materialise when we introduce randomness into an otherwise regular, locally-connected network. These random links provide shortcuts, thus reducing the average distance in the network and eventually leading to short paths between most vertex pairs (small-world phenomenon, which is dubbed 'six degrees of separation' in some popular accounts). Scale-free networks, on the other hand, typically result when randomness in network connectivity is reduced (e.g. via preferential attachment of new nodes).

The literature on small-world and scale-free network phenomena is quite extensive. Following the introduction of the basic concepts and derivation of fundamental properties within the physics and natural sciences communities, the importance of these concepts in computer and control engineering, as well as in computer science, was recognised. This recognition led to numerous studies of real and potential applications in network stability and synchronisation, information diffusion and vulnerability to attacks of various kinds to bring these notions to the attention of engineers and computer scientists (e.g. Wang and Chen 2002; Li and Chen 2003; Li, Wang, and Chen 2004; Chakrabarti and Faloutsos 2006; Griffin and Brooks 2006; Li and Wang 2006; Deng and Zhang 2007; Xia and Hill 2008).

As mentioned in Section 1, our focus in this article is on scale-free networks. In a scale-free network, the fraction $P_{d}(k)$ of vertices having degree $k$, that is, with $k$ connections, asymptotically equals $k^{-\gamma}$, where the characteristic constant $\gamma$ is typically between 2 and 3 , although on occasion it may lie outside these bounds. 
Despite this rather strong common characteristic, there is no unique model for such networks, as many connectivity patterns and growth rules lead to scale-freedom. This is why laws applying to scale-free and other types of complex networks are often approximate. One cannot say with certainty that a particular property will be observed in all scale-free networks, or even in all previously discovered complex networks of a particular kind. Hence, the prevalence of qualifiers such as 'most', 'a vast majority' and 'nearly all' in characterising the degree to which a given generic property is observed in natural or synthetic scale-free networks.

Far from being useless, such imprecise statements still allow us to study and make practically important deductions about various network classes, despite the fact that we cannot characterise them with mathematical precision. In fact, two of the authors have previously proposed deterministic models for certain complex networks (Xiao and Parhami 2006) that allow more precise characterisations. Note, however, that this approach simply replaces imprecision in the discussion of properties with inexactness at the modelling stage. In other words, we can derive very precise results about the deterministic model, but the model is only an approximate representation of real networks of interest.

Given the broad interest in scale-free networks as facilitators of scientific inquiries and tools in a number of important practical applications, the questions of identifying, relating and categorising such networks arise. For example, it would be quite helpful to find simple and quick ways of telling whether a network is scale-free (sufficient conditions) or for ruling out scale-freedom (necessary conditions). In the latter case, the necessary conditions can be used to rule out scale-freedom in a vast majority of networks encountered in practice, thus obviating the need for more extensive and computationally more costly investigation. We offer such a necessary condition in this article. Our proposed condition, that is the multiplicity of degree-1 vertices in the graph, is easily verified based on the static network structure (its topology or connectivity). While the running time for verifying our condition varies with the specific graph representation, it will never exceed $O\left(n^{2}\right)$ for an $n$-vertex graph and is usually as low as $O(n)$, the least possible. As a by-product of our results, we have been able to characterise the distance distribution in scale-free networks, which is a result of interest in its own right.

The rest of this article is organised as follows. After reviewing the parameters and key attributes of scale-free complex networks in Section 3, we provide characteristic conditions for such networks and show that these conditions are both easy to verify and satisfied by many natural and man-made scale-free networks (Section 4). Section 5 is devoted to an analysis of distance distributions in scale-free networks based on the results of Section 4. Section 6 contains our conclusion and some directions for further research.

\section{Scale-free networks}

A graph $G=(V, E)$ has a number of local and aggregate parameters that characterise its structure (regularity, modularity, dimension), connectivity (density, ease/speed of diffusion) and robustness (resilience to random or malicious faults). The parameters that are of interest in this article appear in the following list. Throughout this article, we use ' $\approx$ ' to denote comparableness in terms of the order of magnitude; that is, $A \approx B$ is synonymous with $A=\Omega(B)$.

$$
\begin{aligned}
M & \text { Number of edges; } M=|E| \\
N & \text { Number of vertices; } N=|V| \\
d(v) & \text { Degree of the vertex } v \in V \\
\bar{d} & \text { Average vertex degree of the network; } \\
& \bar{d}=\Sigma_{v \in V} d(v) / N \\
n_{k} & \text { Number of degree- } k \text { vertices; } n_{k}= \\
& |\{v \mid d(v)=k\}|
\end{aligned}
$$

$\delta(u, v) \quad$ Distance between vertices $u$ and $v$

$D \quad$ Network diameter; $D=\max _{u, v \in V} \delta(u, v)$

$\bar{\delta} \quad$ Average distance; $\bar{\delta}=\Sigma_{u, v \in V} \delta(u, v) / N^{2}[\mathrm{We}$ use this more convenient definition, rather than excluding the case $u=v$ and dividing the sum of distances by $N(N-1)$ ]

$q_{k} \quad$ Number of node pairs that are of distance $k$; $q_{k}=|\{(u, v) \mid \delta(u, v)=k\}|$

$P_{d}(k)$ Degree distribution, or fraction of vertices that are of degree $k ; P_{d}(k)=n_{k} / N$ [Probability that a randomly chosen vertex is of degree $k]$

$P_{\delta}(k)$ Distance distribution, or fraction of vertex pairs at distance $k ; P_{\delta}(k)=q_{k} / N^{2}$ [Probability that the distance between a randomly chosen vertex pair is $k$ ]

For many complex networks, the probability distribution $P_{d}(k)$ of the number of degree- $k$ vertices, also known as the degree distribution, can be represented (independent of scale, hence the designation 'scale-free') by a power law with characteristic exponent $\gamma$ :

$$
P_{d}(k)=k^{-\gamma} / c
$$

The term $c$ in the denominator of Equation (1) is a normalising constant, defined as $c=\Sigma_{k \in K} k^{-\gamma}$, where $K$ is the set of all node degrees occurring in the network.

Equation (1) holds in an approximate manner, often providing greater precision for node degrees that are neither too small nor too large. 
Nevertheless, network attributes predicted when Equation (1) is deemed to be exact often match remarkably well with the corresponding attributes of real complex networks. The characteristic exponent $\gamma$ is observed to be in the range 2-3 for most actual complex networks.

Certain network attributes can be derived from Equation (1). For example, the high resilience of scale-free networks in the face of random node failures and their vulnerability to deliberate attacks against selected nodes are consequences of Equation (1), with certain mild assumptions. The fact that scale-free networks exhibit the small-world phenomenon, with the attendant high-clustering coefficients, can be deduced likewise. Interestingly, the small-world phenomenon persists, even if distances are not measured along shortest paths (a feature that would require global knowledge for routing decisions, which is not likely to be available in practice), but rather according to a near-optimal routing strategy with localised decisions (Kim, Yoon, Han, and Jeong 2002). Table 1 lists the parameters $N, M, \bar{d}$ and $\gamma$ for several real scale-free networks (Albert and Barabási 2002; Newman 2003). It demonstrates that scale-free

Table 1. Number of vertices $(N)$, number of edges $(M)$, average degree $(\bar{d})$ and characteristic exponent $(\gamma)$ for some complex networks.

\begin{tabular}{lrrrc}
\hline Network & \multicolumn{1}{c}{$N$} & \multicolumn{1}{c}{$M$} & \multicolumn{1}{c}{$\bar{d}$} & $\gamma$ \\
\hline Internet & 10,687 & 31,992 & 5.98 & 2.5 \\
Film actors & 449,913 & $25,516,482$ & 113.43 & 2.3 \\
Metabolic network & 765 & 3686 & 9.64 & 2.2 \\
Protein interactions & 2115 & 2240 & 2.12 & 2.4 \\
\hline
\end{tabular}

networks with $2 \leq \gamma \leq 3$ are of diverse types that include technological, social and natural networks. The parameters listed are also relevant to our study of distance distribution in Section 5. An augmentation to this table will be presented later on (see Table 2).

\section{Necessary conditions for scale-freedom}

In this section, we study the conditions for vertex degrees of complex networks having scale-free power-law distribution. We assume that the network is connected; similar arguments apply to disconnected networks. Recall that $P_{d}(k)$ stands for the probability distribution of the number of degree- $k$ vertices, $\bar{d}$ represents the average vertex degree and $n_{k}$ denotes the number of vertices of degree $k$. We have $M=1 / 2 N \bar{d}$ and $n_{k}=N P_{d}(k)$, where $N$ and $M$ are number of vertices and edges, respectively. The preceding definitions imply that

$$
\begin{gathered}
\sum_{k=1}^{N-1} n_{k}=N \\
\sum_{k=1}^{N-1} k n_{k}=2 M
\end{gathered}
$$

Supposing that $n_{1} \neq 0$, a condition that is almost guaranteed to be satisfied for actual networks with large $N$, we have $n_{k}=n_{1} P_{d}(k) / P_{d}(1)$ and

$$
\begin{aligned}
\sum_{k=1}^{N-1}\left[P_{d}(k) / P_{d}(1)\right] & =N / n_{1} \\
\sum_{k=1}^{N-1}\left[k P_{d}(k) / P_{d}(1)\right] & =2 M / n_{1}
\end{aligned}
$$

\begin{tabular}{|c|c|c|c|c|c|c|c|}
\hline Network & Type & $N$ & $M$ & $n_{1}$ & $\bar{d}$ & $n_{1} / N$ & $\bar{d} / \log N$ \\
\hline Elegans & Bio & 314 & 363 & 203 & 2.312 & 0.646 & 0.279 \\
\hline Grassland & Bio & 88 & 137 & 23 & 3.114 & 0.261 & 0.482 \\
\hline Helicon & Bio & 710 & 1396 & 267 & 3.932 & 0.376 & 0.415 \\
\hline Mouse & Bio & 13 & 12 & 7 & 1.846 & 0.538 & 0.499 \\
\hline Silwood & Bio & 153 & 365 & 55 & 4.771 & 0.359 & 0.657 \\
\hline Ythan & Bio & 135 & 596 & 7 & 8.830 & 0.052 & 1.248 \\
\hline NCSTRL & $\mathrm{SC}$ & 6396 & 15,872 & 894 & 4.963 & 0.140 & 0.393 \\
\hline AbiWord & SW & 1035 & 1719 & 447 & 3.322 & 0.432 & 0.332 \\
\hline Digital Material & SW & 162 & 252 & 59 & 3.111 & 0.364 & 0.424 \\
\hline Linux & SW & 5285 & 11,352 & 1200 & 4.296 & 0.227 & 0.347 \\
\hline MySQL & SW & 1480 & 4190 & 258 & 5.662 & 0.174 & 0.538 \\
\hline VTK & SW & 771 & 1357 & 348 & 3.520 & 0.451 & 0.367 \\
\hline XMMS & SW & 971 & 1802 & 300 & 3.712 & 0.309 & 0.374 \\
\hline
\end{tabular}

Table 2. Relationship of $n_{1}$ versus $N$ and $\bar{d}$ versus $\log N$, in a number of example networks of biological (Bio), scientific collaboration (SC) and software (SW) types.

Notes: For data on biological networks, see: www.cosin.org/extra/data. Data for NCSTRL was supplied by E.J. Newman. For software networks, see: www.tc.cornell.edu/ myers/Data/SoftwareGraphs/index.html. 
For scale-free networks, we have $P_{d}(k)=P_{d}(1) k^{-\gamma}$, which leads to

$$
\sum_{k=1}^{N-1} k^{-\gamma}=N / n_{1}
$$

Therefore, using the assumption $\gamma \geq 2$, a condition which is known to hold for many scale-free networks (Albert and Barabási 2002; Newman 2003), we have

$$
N / n_{1} \leq \sum_{k=1}^{\infty} k^{-\gamma} \leq \sum_{k=1}^{\infty} k^{-2}=\pi^{2} / 6
$$

This leads to the result $N \approx n_{1}$. Let $f(k)=P_{d}(k) /$ $P_{d}(1)=k^{-\gamma}$. When $N \approx n_{1}$, Equation (3) yields

$$
\begin{aligned}
& \sum_{k=1}^{N-1} f(k) \approx 1 \\
& \sum_{k=1}^{N-1} k f(k) \approx \bar{d}
\end{aligned}
$$

Taking all logarithms to be in base 2, and in view of the assumption $\gamma \geq 2$, Equation (6) yields $1<\bar{d} \leq \log N$, given that $\sum_{k=1}^{N} k^{-1} \approx \log N$. Thus, we have proved that for many real complex networks of scale-free power-law distribution with $\gamma \geq 2$, the number of degree-1 vertices, when nonzero, is of the same order as the network size $N$ and that the average degree is of order $\log N$. Furthermore, if $\log \log N \leq \bar{d} \leq \log N$, then Equation (6) implies in many cases that

$$
f(k) \approx k^{-\gamma}
$$

Elaborating on Equation (7), three canonical cases can be distinguished for the function $f(k)$ when $\log \log N \leq \bar{d} \leq \log N$ : (a) $f(1)=1$ and $f(k)$ a constant for $k \neq 1$, (b) $f(k)=e^{1-k}$ and (c) $f(k) \approx k^{-\gamma}$. For case (c), Equation (6) holds when $2 \leq \gamma<3$. However, for cases (a) and (b), Equation (6b) is not satisfied if Equation (6a) holds. Note that more complex functions satisfying Equation (6) exist. For example, one can define the following function which satisfies Equation (6):

$$
f(k) \approx e^{1-k}, \quad \text { if } k \leq \log N ; \quad f(k) \approx k^{-\gamma}, \text { otherwise }
$$

As a consequence of our above results, the scale-free property of complex networks must be viewed as an approximate or fuzzy property.

Referring to Table 1, which lists the parameters $N$, $M, \bar{d}$ and $\gamma$ for several real scale-free networks, we note that $\bar{d} \approx 1 / 2 \log N$ (respectively, $5 \log N, \log N$ or $\log \log N$ ) for the Internet (film actors, metabolic or protein interaction) network. A wider range of examples from the domains of biological networks, a particular instance of scientific collaboration networks and various types of software graphs appear in Table 2. Recall that $N, M, n_{1}$ and $\bar{d}$ are, respectively, number of vertices, number of edges, number of degree-1 vertices and average vertex degree in the network, and that logarithms are in base 2 .

The data presented in Table 2 allow further evaluation and verification of our results and the associated points discussed above. Note that in all these examples, $n_{1}$ is a sizable fraction of $N$. The variation in $n_{1} / N$ in the sample of Table 2 is approximately an order of magnitude (a factor of about 12.4, to be more precise), while $\bar{d} / \log N$ has a three times narrower variation range. We do not know all the $\gamma$ values for these networks, but the Ythan network, with worst match to our results has $\gamma=1.04$, well below the lower limit of 2 for which we have proved our results.

For many real scale-free networks (Albert and Barabási 2002; Newman 2003), the exponent $\gamma$ satisfies $\gamma<3$. We now proceed to show that this property also follows from our analysis. A connected scale-free network has $\gamma<3$ when $n_{1} \geq 1$ and $N \geq 6$. In fact, for $\gamma \geq 3$, we have

$$
M=\left(n_{1} / 2\right) \sum_{k=1}^{N-1} k^{1-\gamma} \leq\left(\pi^{2} / 12\right) n_{1}<(5 / 6) n_{1}
$$

We see that the assumption $\gamma \geq 3$ leads to the impossible result $N-M>N / 6 \geq 1$.

\section{Distance distribution}

Using the method of Section 4, we can obtain other interesting results on the distance distribution of complex networks. Recall that $D$ and $\bar{\delta}$ represent the diameter and average distance of a complex network, respectively. Assume that there are $q_{k}$ vertex pairs of distance $k$, where for simplicity we include the pairs $(v, v)$ and count both $(u, v)$ and $(v, u)$ for $u \neq v$; that is, we consider the pairs to be ordered. Then we have

$$
\begin{aligned}
\sum_{k=0}^{D} q k & =N^{2} \\
\sum_{k=1}^{D} k q_{k} & =N^{2} \bar{\delta}
\end{aligned}
$$

Clearly, $q_{1}=2 M$. For scale-free networks with $\gamma \geq 2$, we have $M \leq 1 / 2 N \log N$. These networks are also believed to satisfy $D \leq c \log N$, where $c$ is constant. Now, suppose that $M=1 / 2 N \log N$, $D=\log N, \quad \bar{\delta}=1 / 2 \quad \log N \quad$ and $\quad g(k)=q_{k} \quad / \quad(2 M)$. 
Then, Equation (10) yields

$$
\begin{gathered}
\sum_{k=0}^{\log N} g(k) \approx N / \log N \\
\sum_{k=1}^{\log N} k g(k) \approx N / 2
\end{gathered}
$$

The following identities involving binomial coefficients are well known:

$$
\begin{gathered}
\left(\begin{array}{l}
r \\
0
\end{array}\right)+\left(\begin{array}{l}
r \\
1
\end{array}\right)+\left(\begin{array}{l}
r \\
2
\end{array}\right)+\cdots+\left(\begin{array}{l}
r \\
r
\end{array}\right)=2^{r} \\
\left(\begin{array}{l}
r \\
1
\end{array}\right)+2\left(\begin{array}{l}
r \\
2
\end{array}\right)+3\left(\begin{array}{l}
r \\
3
\end{array}\right)+\cdots+r\left(\begin{array}{l}
r \\
r
\end{array}\right)=r 2^{r-1}
\end{gathered}
$$

By Equation (12), $g(k)=\left(\begin{array}{c}\log N \\ k\end{array}\right) / \log N$ satisfies Equation (11). Hence, $q_{k} \approx N\left(\begin{array}{c}\log N \\ k\end{array}\right)$. Thus, we have

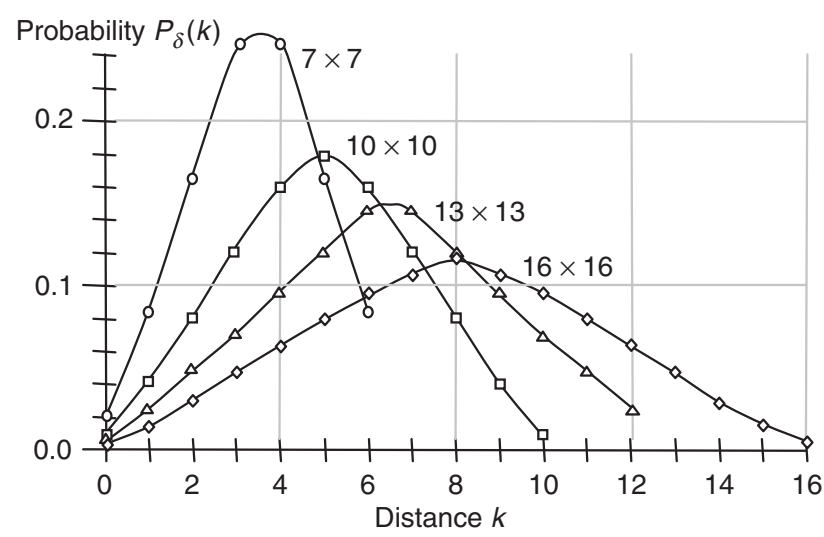

Figure 2. Distance distribution for small, square torus networks.

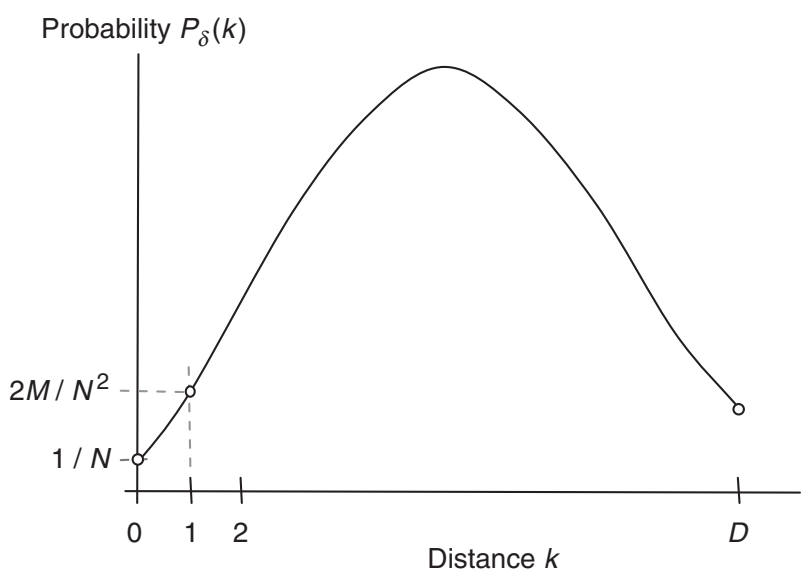

Figure 3. Generic distance distribution in a diameter- $D$ network with $N$ vertices and $M$ edges. obtained the distance distribution for vertex pairs in scale-free complex networks:

$$
P_{\delta}(k)=q_{k} / N^{2} \approx\left(\begin{array}{c}
\log N \\
k
\end{array}\right) / N
$$

One should note that the distance distribution of networks are quite complicated and the formulas derived for $q_{k}$ and $P_{\delta}(k)$ are approximate. In the case of certain regular networks, closed-form expressions for distance distribution can be obtained. For example, the corresponding formulas for $m \times m$ torus networks $\left(N=m^{2}\right)$ are as follows:

$$
q_{0}=N ; \quad q_{k}=q_{m-k}=4 k N(1 \leq k<m / 2) \quad \text { for all } m
$$

$$
q_{m / 2}=(2 m-2) N ; \quad q_{m}=N \quad \text { for } m \text { even }
$$

Many real networks, with which we have experimented, have a distance distribution curve that rises at
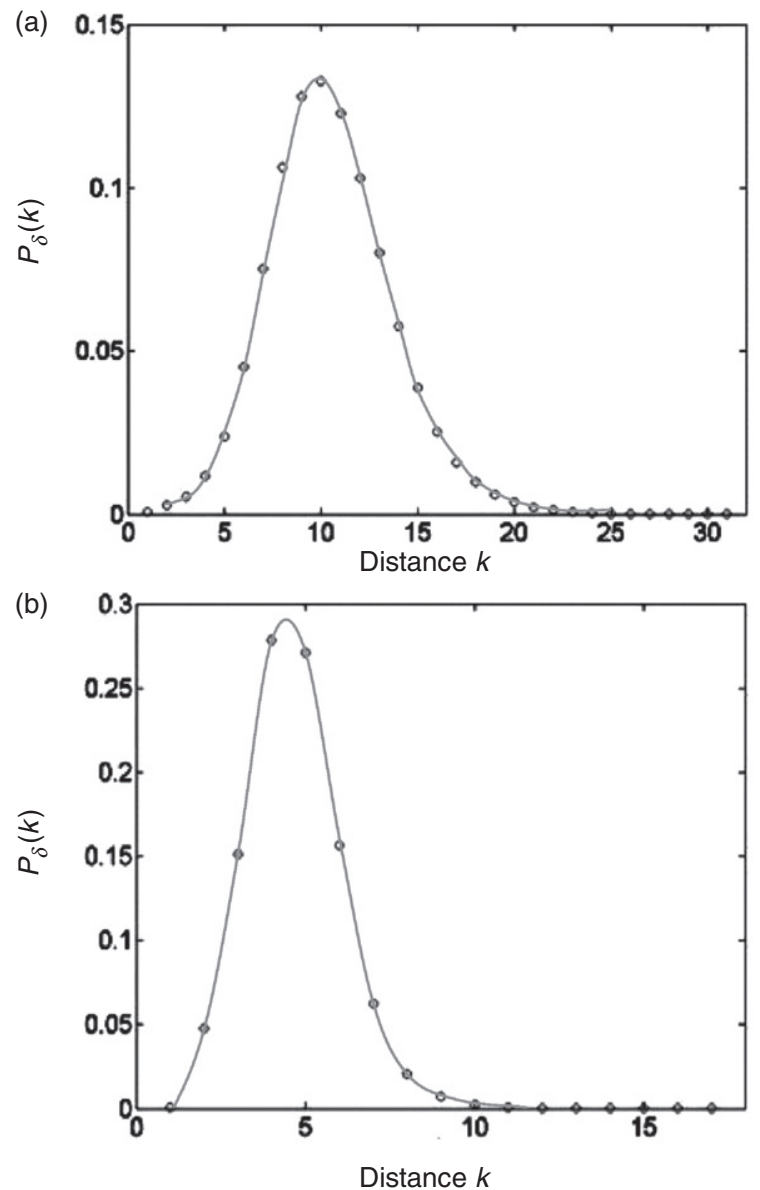

Figure 4. Distance distribution in the maximum component of two actual complex networks: (a) NCSTRL graph (Newman 2001) with 6396 vertices and diameter of 31, and (b) Linux graph (Myers 2003) with 5285 vertices and diameter of 17. 
first, and then exhibits a declining trend, much like those in Figure 2. So, both extremely short and very long distances are less common than values closer to the average. Figure 3 depicts the general form of the distance distribution in complex networks. Figure 4 depicts the distance distributions in two actual complex networks.

\section{Conclusions}

Despite extensive research on large-scale complex networks within multiple scientific disciplines over the past decade, there are still gaping holes in our understanding of such networks (Dorogovtsev and Mendes 2002). New results that shed light on the static structure (topology) and dynamic properties (node interactions) of different classes of large-scale networks are needed to facilitate further progress. We have shown that, when vertex degrees of large networks follow a scale-free power-law distribution with the exponent $\gamma \geq 2$, the number of degree-1 vertices, when nonzero, tends to be of the same order as the network size $N$, and that the average node degree is of order at most $\log N$. Our method can be applied with advantage, because it relies on conditions that are static and easily verified for any network.

We have also derived a closed-form formula approximating the probability that a randomly chosen pair of vertices are at distance $k$ (distance distribution) in scale-free networks. Such distributions are known to be applicable to diverse fields of study, including software architecture and computer communications (Yook, Jeong, and Barabási 2002). Our results on distance distribution are extensible to the case when distances are measured according to a routing strategy with localised decisions (Kim et al. 2002), in lieu of globally optimal routes which are much harder to compute, and thus less likely to be practically useful. Pursuing this and similar extensions constitute fruitful directions for further research.

Our discussion of Section 4 leads to a model for scale-free networks, which are known to satisfy $k^{\gamma}=P_{d}(1) / P_{d}(k)=n_{1} / n_{k}$. Taking this equality to be exact and noting that $n_{1} / n_{k}$ is a rational number, it follows that $k^{\gamma}$ must be an integer that divides $n_{1}$. Consequently, $n_{1}$ must be divisible by the least common multiple of $k_{1}^{\gamma}, k_{2}^{\gamma}, \ldots, k_{l}^{\gamma}$, where $1=k_{1}<k_{2}<\cdots<k_{l}$ is the degree sequence of the network. This yields a general model for scale-free networks that we will aim to study further in the future. Such extensions and variations will further broaden the applications of our results in diverse subfields within computing, communication, biology and the social sciences.

\section{Acknowledgements}

We thank M.E.J. Newman for providing the data for NCSTRL. The research of W.J. Xiao is supported by the Natural Science Foundation of Guangdong Province and by the Foundation of the Guangdong Key Laboratory for Computer Networks.

\section{Notes on contributors}

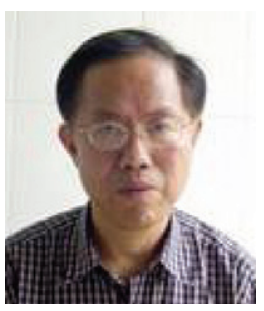

Wenjun Xiao received the $\mathrm{PhD}$ degree in mathematics from Sichuan University, P. R. China, in 1989. Currently, he is a Professor in the School of Computer Science and Engineering, South China University of Technology, Guangzhou, P. R. China. His research interests include discrete mathematics, parallel and distributed computing, complex network and software architecture. He has published more than 60 papers in international conferences and journals, including IEEE Transactions on Computers and IEEE Transactions on Parallel and Distributed Systems on these topics since 1985.

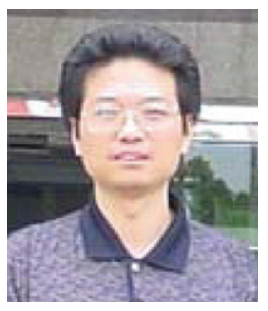

Weidong Chen received the BS degree in mathematics from Huazhong Normal University, Wuhan, P. R. China, in 1991 and the MS degree in computer science from Huazhong University of Science and Technology, Wuhan, P. R. China, in 1997. He is an Associate Professor in the Department of Computer Science at South China Normal University, Guangzhou, P. R. China. He is currently a PhD candidate in computer science at South China University of Technology, Guangzhou, P. R. China. His research interests include graph theory, optimization theory, approximation algorithms and parallel computing.

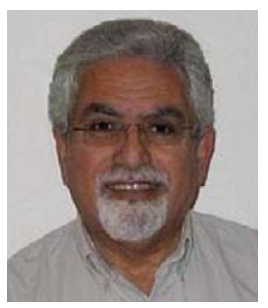

Behrooz Parhami (PhD, University of California, Los Angeles, 1973) is a Professor of Electrical and Computer Engineering at the University of California, Santa Barbara. His research interests include computer arithmetic, parallel processing and dependable computing. In his previous position with Sharif University of Technology in Tehran, Iran (19741988), he was also involved in educational planning, curriculum development, standardization efforts, technology transfer and various editorial responsibilities, including a five-year term as Editor of Computer Report, a Persian language computing periodical. His technical publications include over 260 papers in peer-reviewed journals and international conferences, a Persian-language textbook and an English/ Persian glossary of computing terms. Among his publications are three textbooks on parallel processing (Plenum, 1999), computer architecture (Oxford, 2005) and computer arithmetic (Oxford, 2nd ed., 2010). He is currently serving on the editorial boards of IEEE Transactions on Parallel and Distributed Systems, IEEE Transactions on Computers and 
International Journal of Parallel, Emergent and Distributed Systems. Dr Parhami is a Fellow of both the IEEE and the British Computer Society, a member of the Association for Computing Machinery and a Distinguished Member of the Informatics Society of Iran for which he served as a founding member and President during 1979-1984. He also served as the Chairman of IEEE Iran Section (1977-1986) and received the IEEE Centennial Medal in 1984.

\section{References}

Albert, R., and Barabási, A.-L. (2002), 'Statistical Mechanics of Complex Networks', Reviews of Modern Physics, 74, 47-97.

Barabási, A.-L., and Albert, R. (1999), 'Emergence of Scaling in Random Networks', Science, 286, 509-512.

Barabási, A.-L., and Bonabeau, E. (2003), 'Scale-free Networks', Scientific American, 288, 60-69.

Chakrabarti, D., and Faloutsos, C. (2006), 'Graph Mining: Laws, Generators, and Algorithms', ACM Computing Surveys, 38, A1-A69.

Deng, Z., and Zhang, Y. (2007), 'Collective Behavior of a Small-world Recurrent Neural System with Scale-free Distribution', IEEE Transactions on Neural Networks, 18, 1364-1375.

Dorogovtsev, S.N., and Mendes, J.F.F. (2002), 'Evolution of Networks', Advances in Physics, 51, 1070-1187.

Griffin, C., and Brooks, R. (2006), 'A Note on the Spread of Worms in Scale-free Networks', IEEE Transactions on Systems, Man, and Cybernetics - Part B: Cybernetics, 36, 198-202.

Kim, B.J., Yoon, C.N., Han, S.K., and Jeong, H. (2002), 'Path Finding Strategies in Scale-free Networks', Physical Review E, 65, Article 027103.

Li, X., and Chen, G. (2003), 'Synchronization and Desynchronization of Complex Dynamical Networks: An
Engineering Viewpoint', IEEE Transactions on Circuits and Systems - I: Fundamental Theory and Applications, 50, 1381-1390.

Li, X., and Wang, X. (2006), 'Controlling the Spreading in Small-world Evolving Networks: Stability, Oscillation, and Topology', IEEE Transactions on Automatic Control, 51, 534-540.

Li, X., Wang, X., and Chen, G. (2004), 'Pinning a Complex Dynamical Network to Its Equilibrium', IEEE Transactions on Circuits and Systems - I: Regular Papers, 51, 2074-2087.

Myers, C.R. (2003), 'Software Systems as Complex Networks: Structure, Function, and Evolvability of Software Collaboration Graphs', Physical Review E, 68, Article 0461160.

Newman, M.E.J. (2001), 'Scientific Collaboration Networks - I. Network Construction and Fundamental Results', Physical Review E, 64, Article 016131.

Newman, M.E.J. (2003), 'The Structure and Function of Complex Networks', SIAM Review, 45, 167-256.

Wang, X.F., and Chen, G. (2002), 'Synchronization in Scale-free Dynamical Networks: Robustness and Fragility', IEEE Transactions on Circuits and Systems - I: Fundamental Theory and Applications, 49, 54-62.

Watts, D.J., and Strogatz, S.H. (1998), 'Collective Dynamics of "Small-world" Networks', Nature, 393, 440-442.

Xia, Y., and Hill, D.J. (2008), 'Attack Vulnerability in Complex Communication Networks', IEEE Transactions on Circuits and Systems - II: Express Briefs, $55,65-69$.

Xiao, W.J., and Parhami, B. (2006), 'Cayley Graphs as Models of Deterministic Small-world Networks', Information Processing Letters, 97, 115-117.

Yook, S.-H., Jeong, H., and Barabási, A.-L. (2002), 'Modeling the Internet's Large-scale Topology', Proceedings of the US National Academy of Sciences, 99, 13382-13386. 